\title{
Research Paper \\ Comparison of Salivary and Gingival Crevicular Fluid Periostin Levels in Chronic Periodontitis Patients and Healthy Subjects
}

\author{
Fatemeh Momeni ${ }^{1}\left(\mathbb{C}\right.$, Afrooz Nakhostin $^{2}$ (1) ${ }^{*}$ Mojtaba Bayani ${ }^{3}$ (1)
}

1. Student Research Center, Arak University Medical Sciences, Arak, Iran

2. School of Dentistry, Arak University of Medical Sciences, Arak, Iran.

3. Department of Periodontics, Faculty of Dentistry, Arak University of Medical Sciences, Arak, Iran.

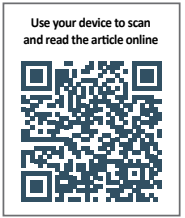

Crtation: Momeni F, Nakhostin A, Bayani M. [Comparison of Salivary and Gingival Crevicular Fluid Periostin Levels in Chronic Periodontitis Patients and Healthy Subjects (Persian)]. Journal of Arak University of Medical Sciences (JAMS). 2020; 23(1):72-81. https://doi.org/10.32598/JAMS.23.1.5710.2

https://doi.org/10.32598/JAMS.23.1.5710.2

Key words:

Chronic periodontitis,

Periostin, Gingival

crevicular fluid, Saliva

\section{A B STRACT}

Background and Aim Periostin acts as necessary protein in tissue development and has a key role in tooth-supporting tissues such as periodontal ligament. The effect of inflammation on reducing periostin level has been shown in some studies. The aim of this study was to compare the salivary and Gingival Crevicular Fluid (GCF) periostin levels in patients with chronic periodontitis and healthy peers.

Methods \& Materials In this matched case-control study, 106 participants (53 patients with chronic periodontitis and 53 healthy controls) were studies after signing a informed consent form. They were matched for age, gender, weight, and Body Mass Index (BMI). The GCF and salivary samples were collected from all participants and were assessed using standard Enzyme-Linked Immunosorbent Assay (ELISA). The statistical analysis was conducted in Stata V. 11.

Ethical Considerations This study was approved by the Research Ethics Committee of Arak University of Medical Sciences (Code: IR.ARAKMU.REC.1397.34).

Results The salivary and GCF periostin levels was significantly lower in patients than in healthy subjects $(\mathrm{P}<0.001)$. Moreover, the periostin levels was significantly different based on periodontal parameters $(\mathrm{P}<0.001)$.

Conclusion There is association between the incidence of chronic periodontitis and salivary and GCF periostin levels. Hence, the periostin may act as a potential biomarker for the diagnosis of chronic periodontitis and prevention of its progression.

\section{Extended Abstract}

\section{Introduction}

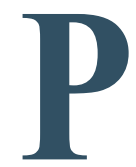

eriodontitis is an inflammatory disorder of the periodontium that affects the tooth-supporting tissues. It is characterized by loss of gingival adhesions and, in more advanced stages of the disease, alveolar bone resorption [1]. Perios- tin is a matriarchal protein secreted by periodontal ligament fibroblasts [3]. The role of periostin in tooth development is very important [4]. In some studies, the study of Gingival Crevicular Fluid (GCF) has also been proposed for measuring the presence of periodontitis [8]. Some studies have reported an association between the severity of periodontitis and periostin level [12-10]. Since contradictory results have reported for the relationship between them and also due to the lack of studies measured both salivary

\section{* Corresponding Author:}

Mojtaba Bayani, PhD.

Address: Department of Periodontics, Faculty of Dentistry, Arak University of Medical Sciences, Arak, Iran.

Tel: +98 (912) 1754901

E-mail: mbayani@mail.com 
and GCF periostin levels, this study aimed to examine GCF and salivary periostin levels in patients with chronic Periodontitis compared to controls.

\section{Materials and Methods}

This matched case-control study was conducted on 53 patients with chronic periodontitis and 53 healthy peers. Three periodontal parameters were evaluated and recorded for each patient: Bleeding on Probing (BOP) [3], Clinical Attachment Level (CAL) [13], and Probing Pocket Depth (PPD). All evaluations were performed by a periodontist. In patients, only one site was selected for sampling, while several sites were selected for sampling in controls to ensure that a sufficient amount of gingival fluid was collected. For sampling, an absorbent paper cone was inserted into the gingival crevice and left in position for one minute. After absorbing the fluid, we slowly removed the paper cone. The salivary sample was collected by using spitting method. All samples were evaluated by ELISA technique. Independent t-test at $95 \%$ confidence interval was used to investigate the difference between the mean levels of periostin in the two groups and also to compare the mean levels of periostin based on periodontal parameters.

\section{Results}

The distribution of age, sex, weight, and body mass index were the same in both groups, and there was no significant difference between them. However, there was a significant difference between the two groups in terms of BOP, CAL, and $\mathrm{PPD}(\mathrm{P}<0.001)$. The mean periostin levels are shown in Table 1 based on periodontal parameters. As can be seen, mean periostin level was significantly higher in case of $\mathrm{BOP}<20 \%, \mathrm{CAL}<1 \mathrm{~mm}$, and $\mathrm{PPD}<3 \mathrm{~mm}(\mathrm{P}<0.001)$. The mean GCF and salivary periostin levels in both groups are presented in Table 2. As can be seen, there is a significant difference in GCF and salivary periostin levels between study groups $(\mathrm{P}<0.001)$.

\section{Discussion}

The study simultaneously measured the GCF and salivary periostin levels of patients with chronic periodontitis compared to the healthy peers. Periostin plays a key role in the development of tooth-supporting tissues [17-15]. In a study conducted by Balli et al., and Rezaei et al., the serum and GCF periostin levels in patients with periodontitis were significantly lower than in the healthy group $[10,18]$. This is consistent with our results. In another study, the GCF periostin levels of patients with chronic and aggressive periodontitis were compared with healthy people. The results showed the high GCF periostin levels of patients compared to control, where it was lower in patients aggressive Periodontitis compared to those with chronic Periodontitis [21]. In our study, both GCF and salivary periostin levels were lower in patients compared to controls. Periostin may act as a potential biomarker for the diagnosis of periodonti-

Table 1. The mean periostin levels based on periodontal parameters

\begin{tabular}{|c|c|c|c|c|}
\hline \multicolumn{2}{|c|}{ Parameters } & \multirow{2}{*}{$\begin{array}{c}\text { Mean }(\mathrm{pg} / \mathrm{mL}) \\
7.42\end{array}$} & \multirow{2}{*}{$\begin{array}{c}\text { SD } \\
1.12\end{array}$} & \multirow[t]{2}{*}{$\mathbf{P}$} \\
\hline \multirow{3}{*}{ BOP } & $<20 \%$ & & & \\
\hline & & & & \multirow[t]{2}{*}{$<0.001$} \\
\hline & $>20 \%$ & 3.71 & 0.98 & \\
\hline \multirow{3}{*}{ CAL } & $<1 \mathrm{~mm}$ & 6.19 & 1.53 & \multirow{3}{*}{$<0.001$} \\
\hline & & & & \\
\hline & $\geq 4 \mathrm{~mm}$ & 2.5 & 1.01 & \\
\hline \multirow{3}{*}{ PPD } & $<3 \mathrm{~mm}$ & 6.12 & 1.49 & \multirow{3}{*}{$<0.001$} \\
\hline & & & & \\
\hline & $\geq 4 \mathrm{~mm}$ & 1.99 & 0.84 & \\
\hline
\end{tabular}

Table 2. The GCF and salivary periostin levels in the study groups

\begin{tabular}{ccccc}
\hline Variable & Samples & Patients & Controls & P \\
\hline Salivary $(\mathrm{pg} / \mathrm{mL})$ & Mean \pm SD & $140.35 \pm 23.7$ & $459.8 \pm 83.5$ & $<0.001$ \\
\hline GCF $(\mathrm{pg} / \mathrm{mL})$ & Mean \pm SD & $10.17 \pm 1.65$ & $29.75 \pm 4.59$ & $<0.001$ \\
\hline
\end{tabular}


tis. Therefore, in practice, it can probably be used to predict and thus prevent the progression of this disease.

\section{Ethical Considerations}

Compliance with ethical guidelines

This study was conducted after obtaining an ethical approval (Code: IR.ARAKMU.REC.1397.3) from the Research Ethics Committee of Arak University of Medical Sciences, and a written consent from the participants.

Funding

This study was financially supported by the Deputy for Research and Technology of Arak University of Medical Sciences.

\section{Authors' contributions}

Scientific design and management: Mojtaba Bayani; Design and implementation: Afrooz Nakhostin; Implementation of practical research process and writing: Mojtaba Bayani, Fatemeh Momeni.

\section{Conflicts of interest}

The authors declare no conflict of interest.

\section{Acknowledgements}

The authors would like to thank the Deputy for Research of Arak University of Medical Sciences for their financial support. 


\title{
بررسى مقايسهای سطح يريوستين در بزاق و مايع شيار لثهاي افراد مبتلا به يريودنتيت مزمن و افراد سالم
}

\author{
فاطمه مومنى '(1)، افروز نخستين' (1)، "مجتبى بيانى \\ ا. مركز تحقيقات دانشجويى دانشعاه علوم يزشكى الراك، اراك، ايران.

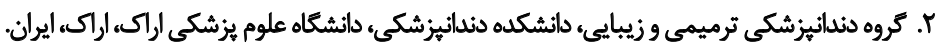

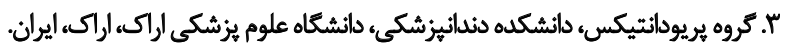

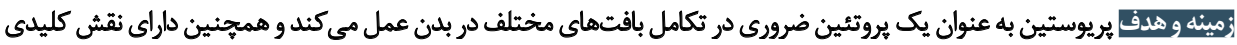

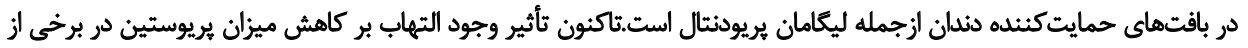

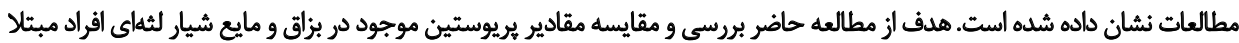

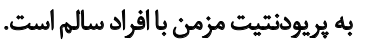

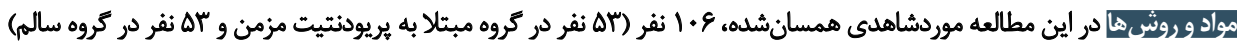

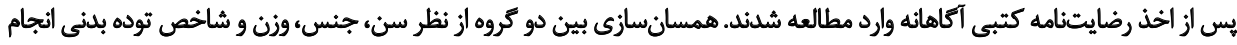

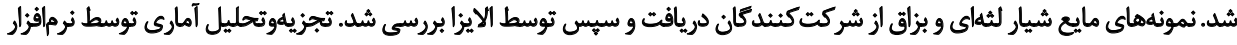
stata v. 11

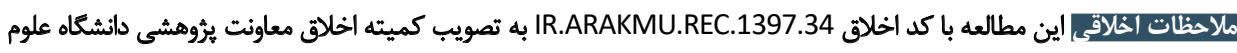

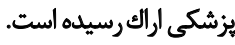

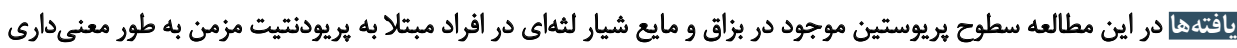

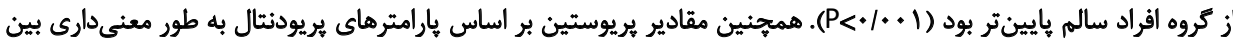

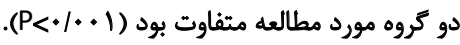

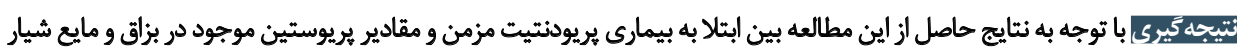

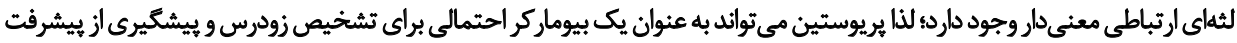

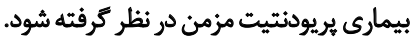

اطلاعات مقاله:

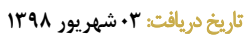

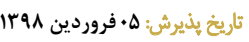

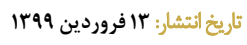

نقش يريوستين در تكامل دندان بسيار كليدى و مهم است و وإِ

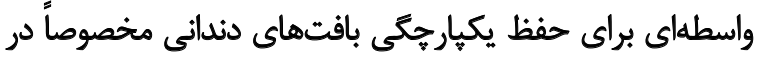
ناحيه تماس بين بافت نرم و سخت دهان است [أ] هيريوستين

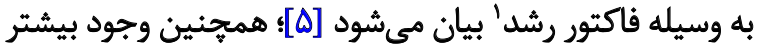

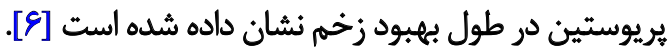
بررسى تركيبات موجود در بزاق ميىتوائد به تشخيص زودهنگام

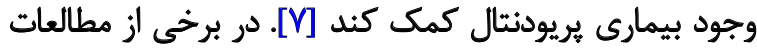

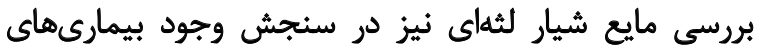

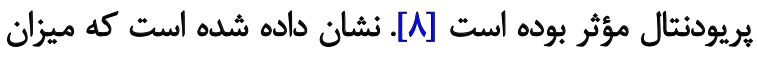

1. Transforming Growth Factor $-\beta$

مقدمه

يريودنتيت مزمن يكى از بيمارى هاي التهابي است كه به وسيله

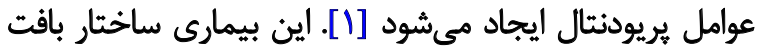

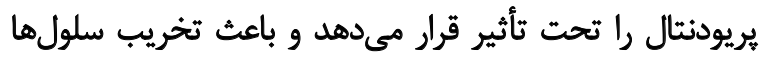

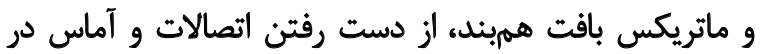

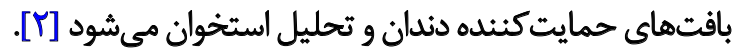
يريوستين يك يروتئين ماتريسلولار است كه به وسيله

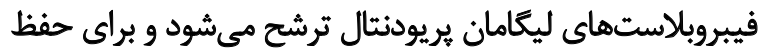

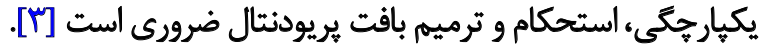
-

ن نويسئده مسئول: مجثبي بياني

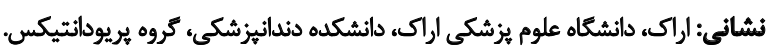

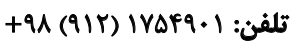
بست الكترونيكى: mbayani@mail.com 
و جاقى (شاخص توده بدنى بيشتر از بهات )، ابتلا به سرطان، ابتلا

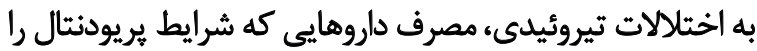

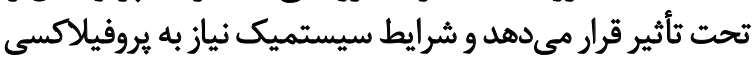
آنتىبيوتيك قبل از معاينه يريونتئال.

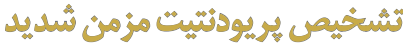

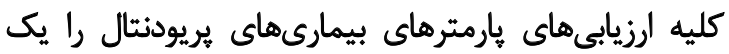

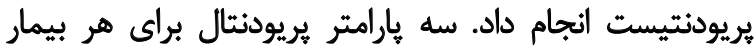

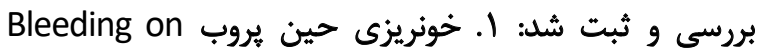
:Probing (BOP)

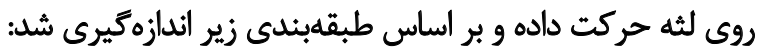

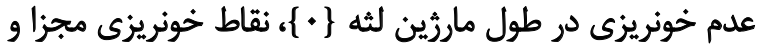

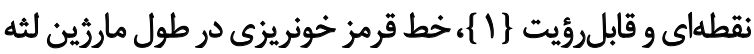

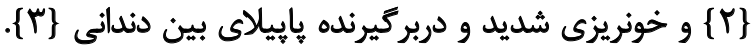

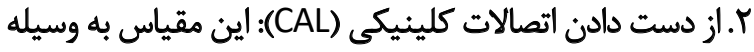

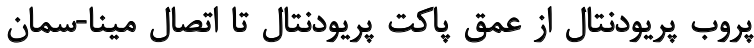
Cemento enamel junction (CEJ)

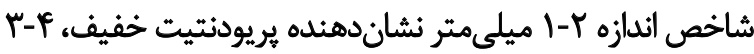

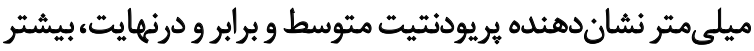

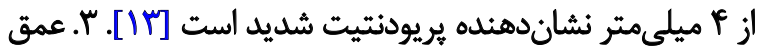

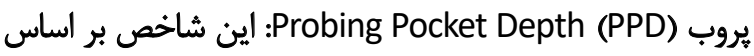

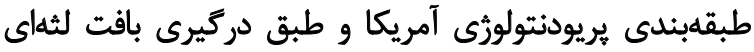

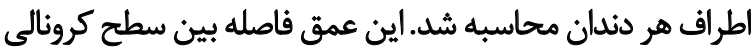

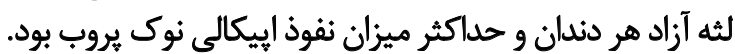

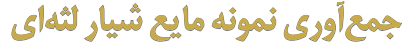

در بيماران مبتلا به يريودنتيت مزمن فقط يك محل براي

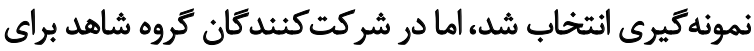

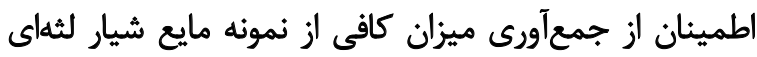

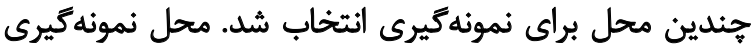

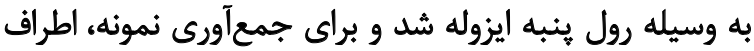

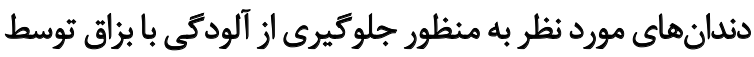

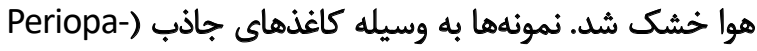
(per, Proflow Inc., Amityville, NY, USA شد. مقدار مايع در هر كاغذ جاذب با استفاده از دستكاه كاليبره PeriotronTM 6000 Proflow Inc., Amityville, NY,) اندازهيرى شد. براى نمونه مقيرى، اين كاغذها در شيار لثه

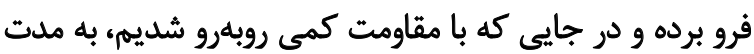

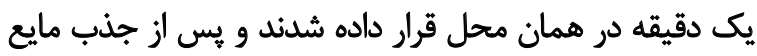

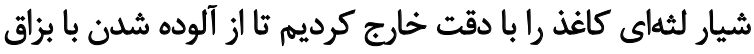

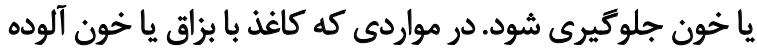

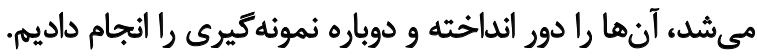

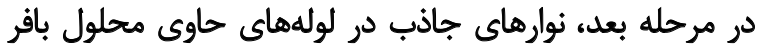

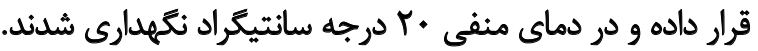

برخى از سايتوكينها در بزاق بيماران مبتلا به يريودنتيت مزمن

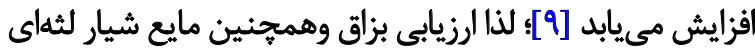

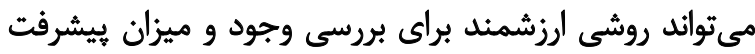
بيمارى هاي يريودنتال باشد.

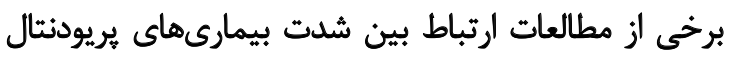

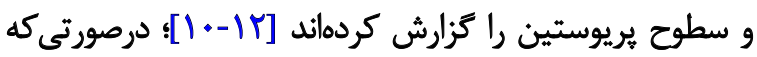

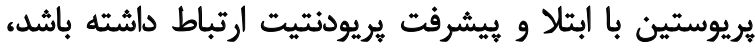

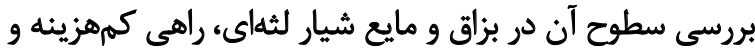

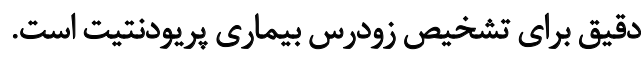

با توجه به نتايج متفاوت در خصوص ارتباط سطوح يريوستين

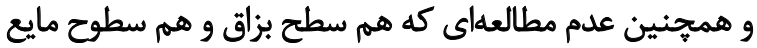

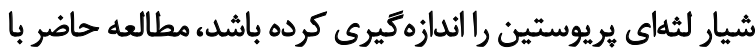

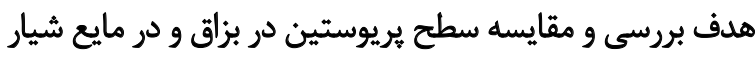

لثهاى افراد مبتلا به يريودنتيت مزمن با برافئ براد سالم انجام شد.

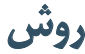

\section{digoi itsos}

اين مطالعه موردشاهدى همسانشده روى \& 1 نفر شامل يك

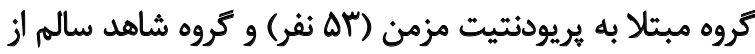

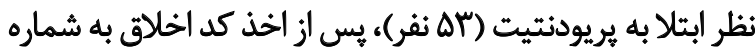

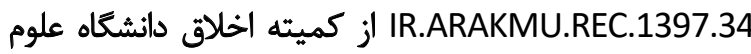

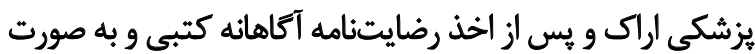

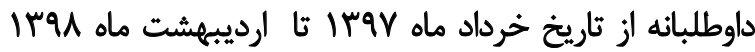

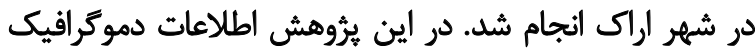

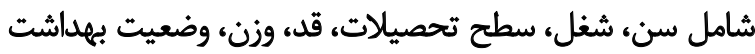

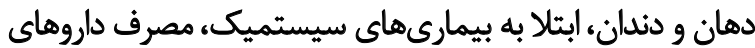

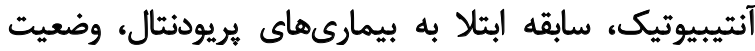

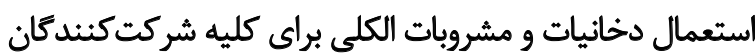

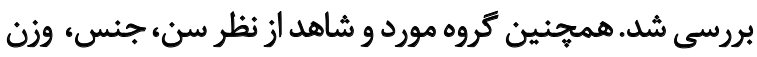

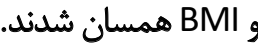

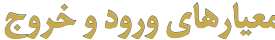

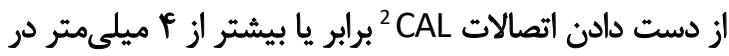

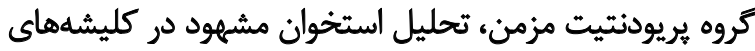

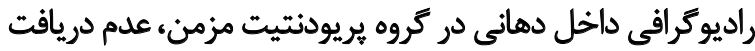

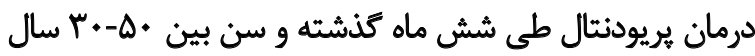

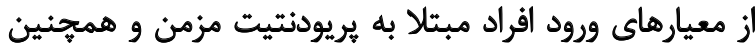

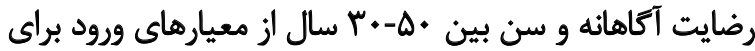

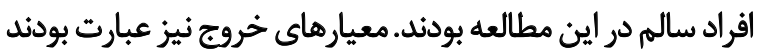

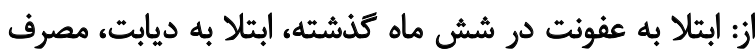

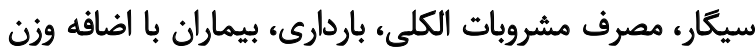

2.Clinical Attachment Loss 
متغيرها بين دو گروه همسان شدهاند، توزيع فراوانى سن، جنس،

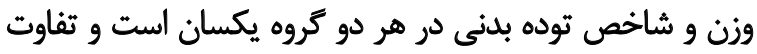

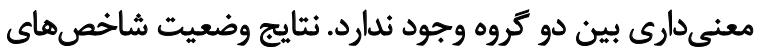

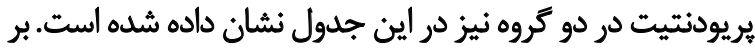

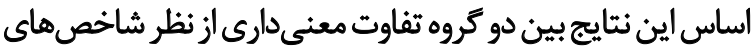

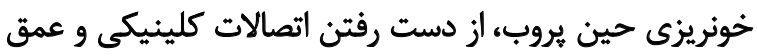

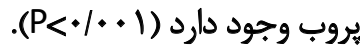

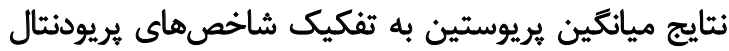

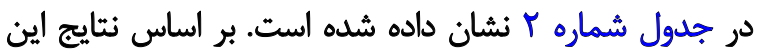

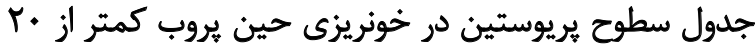

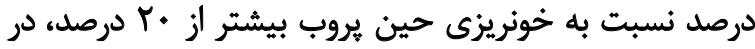

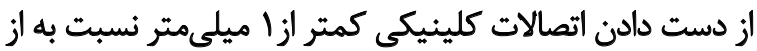

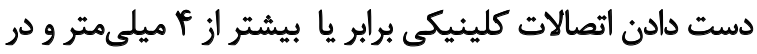

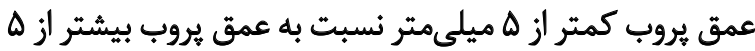

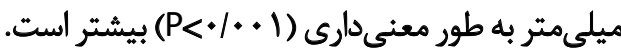

نتايج ميانگين سطوح بريوستين در نمونه بزاق و مايع شيار

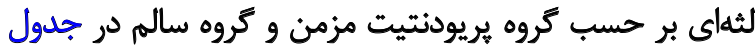

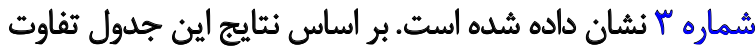

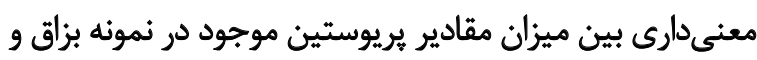

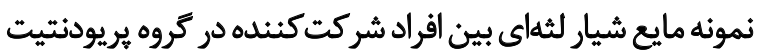

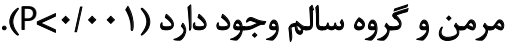

بحث

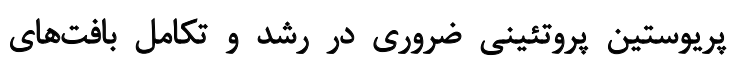

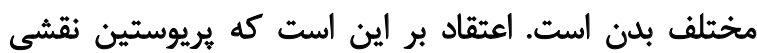

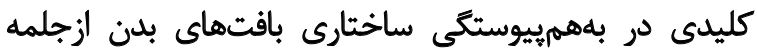

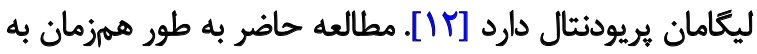

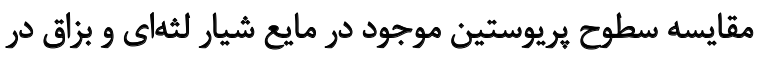

مقادير بريوستين موجود در مايع شيار لثهاي با استفاده از تست

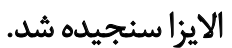

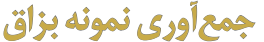

نمونه بزاق با استفاده از روش تف كردن بزاق توسط بيمار در لوله

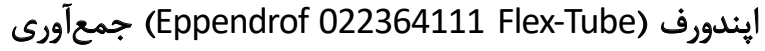

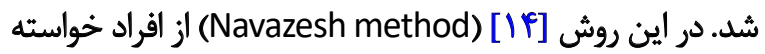

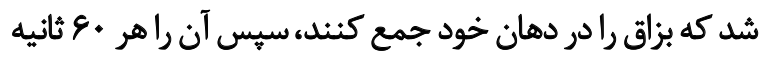

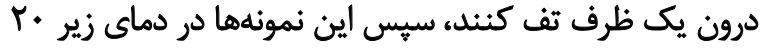

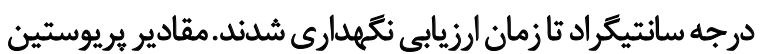

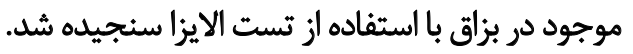

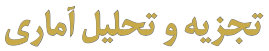

براى بررسى توزيع فراوانى از آزمونهاى توصيفى نظير ميانكين،

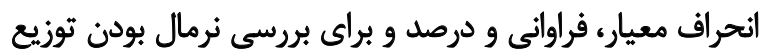

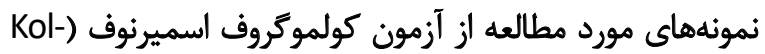
(mogorov Smirnov سطوح بريوستين در دو كروه و همجينين براي مقايسه ميانكين براين

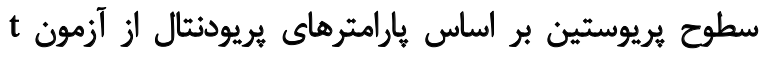

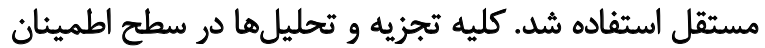
هو درصد و با استفاده از نرمافزار آمارى Stata V. 11 انجام شد.

ثنايج

نتايج آزمون كولموكروف اسميرنوف براى سنجش نرمال بودن

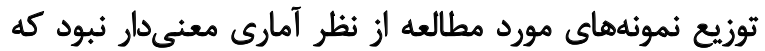

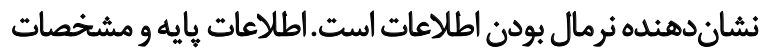

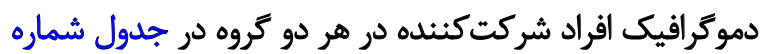

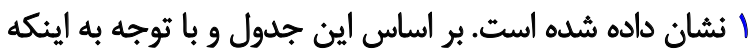

جدول ا. اطلاعات بايه افراد شركت كنيده

\begin{tabular}{|c|c|c|c|c|}
\hline$P$ & تئروه سالم & كروه يريودنتيث مزمن & & \\
\hline- & 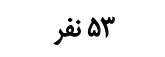 & ماه نفر & & تعلاد كل \\
\hline.$/ m r^{r}$ & RE/TV & $r e / T V$ & 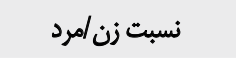 & جنس (تعداد) \\
\hline - larr & $\begin{array}{c}r q / u \pm r / q r \\
\Delta \cdot / r\end{array}$ & $\begin{array}{c}f+/ r p \pm F / M 1 \\
r q / r 1\end{array}$ & ميائكين土|نحراف معيار & سن (سال) \\
\hline.$|f i|$ & $V / M P \pm Y / M$ & ENA \pm Y/N & ميائكين +انحراف معيار & 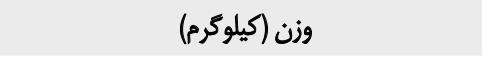 \\
\hline$\cdot / p r r$ & 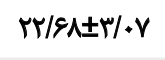 & $r r / / V \pm r / T \Delta$ & ميائكين +انحراف معيار & شاخص توده بدنى (BMI) (كيلوكرم/مترمربح) \\
\hline$<\cdot 1 *+1$ & $\Delta / r m \pm+18 \Delta$ & $r / \Delta \pm \varphi / \Delta \Delta$ & ميانكين ثلانحراف معيار & خونريزى حين يروب (BOP) (درصد) \\
\hline$<\cdot 1 \cdot .1$ & $\cdot / v a \pm \cdot / m$ & P/qY $\pm 1 / 1 r$ & ميانكين لانحراف معيار & از دست دادن اتصالات كلينيكي (CAL) (ميلىمتر) \\
\hline$<* 1 \cdot+1$ & $1 / \cdot 9 \pm \cdot / 199$ & $\Delta / q r \pm I / r q$ & ميانكين ثانحر اف معيار & عمق يروب (PPD) (ميلىمتر) \\
\hline
\end{tabular}


جدول r. بررسى ارتباط سطوح بريوستين با ساير متغيرهاى موجود در مطالعه

\begin{tabular}{|c|c|c|c|c|}
\hline $\mathbf{P}$ & انحراف معيار & 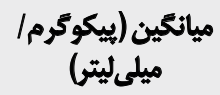 & \multicolumn{2}{|c|}{ متغير } \\
\hline \multirow{2}{*}{$<+1+\infty 1$} & I/K & V/AT & كمتر از •ب درصد لثه اطراف دثلانهايى موجود & \multirow{2}{*}{ خونيزى حين بروب (درصل) } \\
\hline &.$/ 4$ & $r M$ & بيشتر از • بدرصد ليه اطراف دندانهاي هوجود & \\
\hline \multirow{2}{*}{$<+1++1$} & var & $8 / 19$ & كمثر از اميلىمتر & \multirow{2}{*}{ زز دست دادن اتصالات كلينيكى (ميلىمتر) } \\
\hline & $1 / .1$ & $r / \Delta$ & برابر يا بيشتر از f ميلىمتر & \\
\hline \multirow{2}{*}{$<+1+.1$} & $1 / p 9$ & $\sin$ & كمتر از بَ ميلمىمتر & \multirow{2}{*}{ عمق يروب (ميلىمتر) } \\
\hline & . /Ar & 1/99 & برابر يا بيشتر ازو ميلى متر & \\
\hline
\end{tabular}

جدول ثل. ميانتين يريوستين موجود در بزاق و مايع شيار لثهاى در دو كروه مورد و شاهد

\begin{tabular}{|c|c|c|c|c|}
\hline $\mathbf{P}$ & مَروه سالم, & كروه يريودنتيت مزمن & 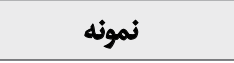 & مثغير \\
\hline$<+. . .1$ & $P \Delta q / A \pm \Lambda r / \Delta$ & $1 f=/ r \Delta \pm \pi / N$ & هيانكين لانحراف معيار & بزاق (ييكوكرم/ميليليتي) \\
\hline$<+. .1$ & $r q / v \Delta \pm r / \Delta q$ & $1 . / / \vee \pm V / / 9 \Delta$ & ميانكين ثلانحراف معيار & مايع شيار لثهاي (ييكوكرم/ميلىليتر) \\
\hline
\end{tabular}

ليكامان بريودنتال در خلال بيمارىهاى لثه است كه ساخت

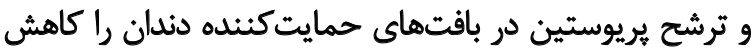

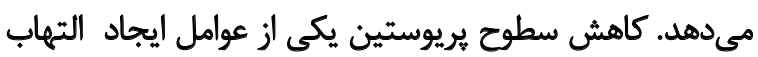

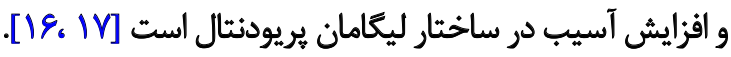

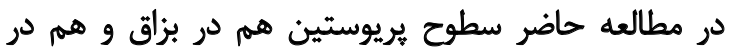

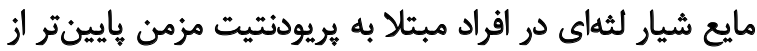

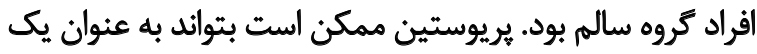

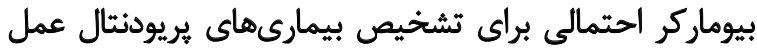

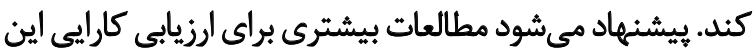

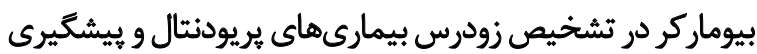

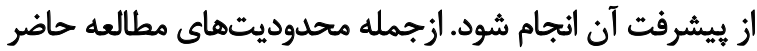

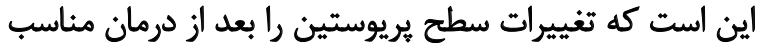

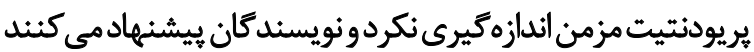

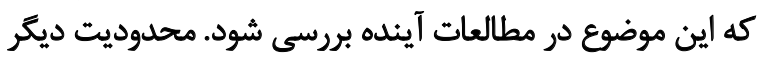

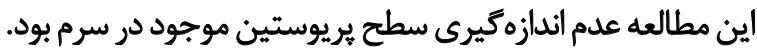

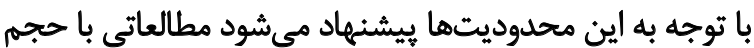

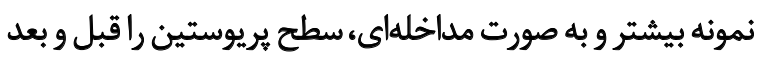
از درمان يريودنتيث مزمن اندازهيرى و مقايسه كنيند.

\section{نتيجهَّيرى}

با وجود اين محدوديتها نتايج مطالعه حاضر تواه خوبى براى

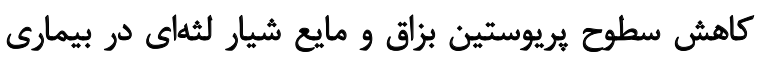

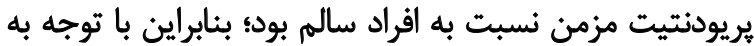

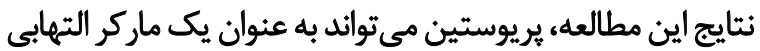

فراد مبتلا به بيمارى هريودنتيت مزمن در مقايسه با كروه سالم

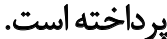

يريوستين نقشى كليدى در تكامل بافتهاى حمايت كننده

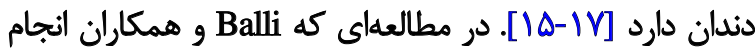

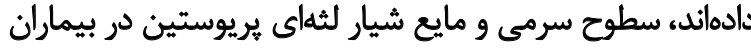

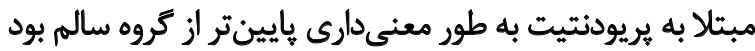

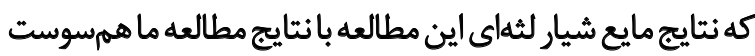

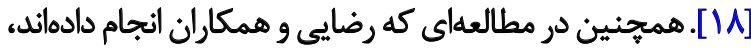

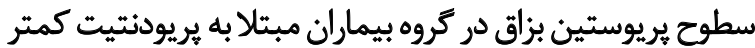

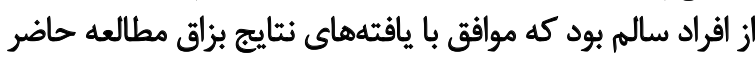

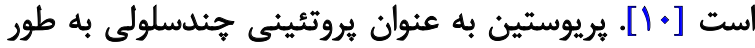

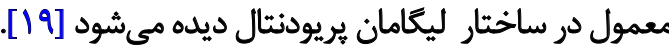
روهاسازى يريوستين را در فيبروبلاستهاى لثهاي

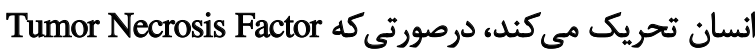

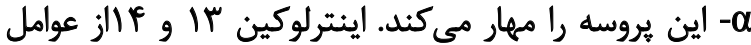

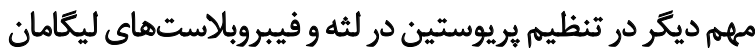

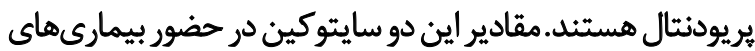

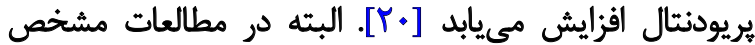

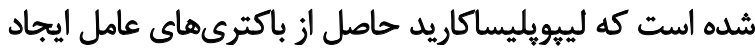

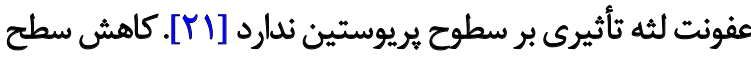

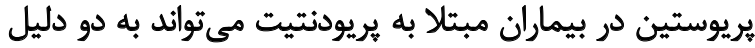

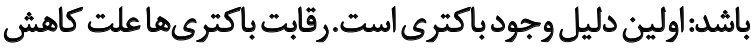

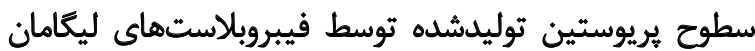

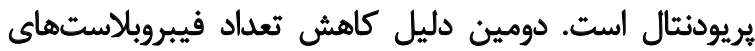


مناسب براى تشخيص زودرس هريودنتيت مزمن و ييشكيرى از يميشرفت آن در نظر كرفته شود.

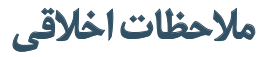

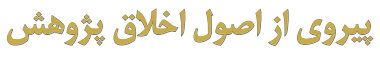

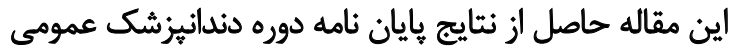

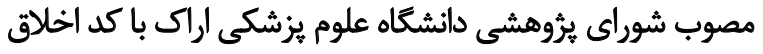
IR.ARAKMU.REC.1397.34 است. است.

$$
\text { Sto collo }
$$

معاونت محترم تحقيقات و فناورى دانشكاه علوم يزشكى اراى تأمين مالى اين يرورثه را بر عهده داشتئه است.

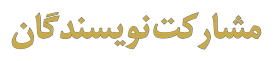

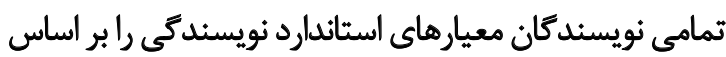

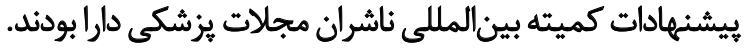

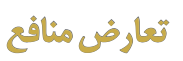

بدينوسيله نويسندكان تصريح مى كنند كه هيج كونه تضاد منافعى در خصوص يزوهش حاضر وجود ندارد.

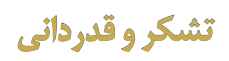

اين مطالعه هيجّونه حامى مالى نداشته است. بدينوسيله از ازئ

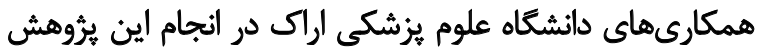

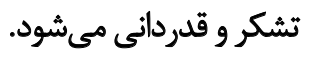




\section{References}

[1] Kinane DF, Stathopoulou PG, Papapanou PN. Periodontal diseases. Nat Rev Dis Primers. 2017; 3:17038. [DOI:10.1038/nrdp.2017.38] [PMID]

[2] Scannapieco FA. Periodontal inflammation: From gingivitis to systemic disease? Compend Contin Educ Dent. 2004; 25(7 Suppl. 1):16-25. [PMID]

[3] Takayama I, Tanabe H, Nishiyama T, Ito H, Amizuka N, Li M, et al. Periostin is required for matricellular localization of CCN3 in periodontal ligament of mice. J Cell Commun Signal. 2017; 11(1):5-13. [DOI:10.1007/ s12079-016-0371-5] [PMID] [PMCID]

[4] Du J, Li M. Functions of periostin in dental tissues and its role in periodontal tissue regeneration. Adv Exp Med Biol. 2019; 1132:63-72. [DOI:10.1007/978-981-13-6657-4_7] [PMID]

[5] Ouanouki A, Lamy S, Annabi B. Periostin, a signal transduction intermediate in TGF- $\beta$-induced EMT in U-87MG human glioblastoma cells, and its inhibition by anthocyanidins. Oncotarget. 2018; 9(31):22023. [DOI:10.18632/oncotarget.25153] [PMID] [PMCID]

[6] Walker JT, McLeod K, Kim S, Conway SJ, Hamilton DW. Periostin as a multifunctional modulator of the wound healing response. Cell Tissue Res. 2016; 365(3):453-65. [DOI:10.1007/s00441-016-2426-6] [PMID] [PMCID]

[7] Chomyszyn-Gajewska M. [Evaluation of chosen salivary periodontal disease markers (Polish)]. PrzegI Lek. 2010; 67(3):213-6. [PMID]

[8] Gupta S, Chhina S, Arora SA. A systematic review of biomarkers of gingival crevicular fluid: Their predictive role in diagnosis of periodontal disease status. J Oral Biol Craniofac Res. 2018; 8(2):98-104. [DOI:10.1016/j. jobcr.2018.02.002] [PMID] [PMCID]

[9] Deo V, Bhongade ML. Pathogenesis of periodontitis: Role of cytokines in host response. Dent Today. 2010; 29(9):60-2, 64-6; quiz 68-9. [PMID]

[10] Esfahrood ZR, Vardian ST, Yadegari Z, Adhim M, Saravi NSV. Periostin levels in saliva of patients with chronic periodontitis. J Indian Soc Periodontol. 2018; 22(1):25-7. [DOI:10.10.4103/jisp.jisp_239_17] [PMID] [PMCID]

[11] Kumaresan D, Balasundaram A, Naik VK, Appukuttan DP. Gingival crevicular fluid periostin levels in chronic periodontitis patients following nonsurgical periodontal treatment with low-level laser therapy. Eur J Dent. 2016; 10(4):546-50. [DOI:10.4103/1305-7456.195179] [PMID] [PMCID]

[12] Padial-Molina M, Volk S, Taut A, Giannobile W, Rios H. Periostin is down-regulated during periodontal inflammation. J Dent Res. 2012; 91(11):1078-84. [DOI:10.1177/0022034512459655] [PMID] [PMCID]

[13] Armitage GC. Development of a classification system for periodontal diseases and conditions. Ann Periodontol. 1999; 4(1):1-6. [DOI:10.1902/ annals.1999.4.1.1] [PMID]

[14] Navazesh M. Methods for collecting saliva. Ann N Y Acad Sci. 1993; 694:72-7. [DOI:10.1111/j.1749-6632.1993.tb18343.x] [PMID]

[15] Padial-Molina M, Volk SL, Taut AD, Giannobile WV, Rios HF. Periostin is down-regulated during periodontal inflammation. J Dent Res. 2012; 91(11):1078-84. [DOI:10.1177/0022034512459655] [PMID] [PMCID]

[16] Kruzynska-Frejtag A, Wang J, Maeda M, Rogers R, Krug E, Hoffman $S$, et al. Periostin is expressed within the developing teeth at the sites of epithelial-mesenchymal interaction. Dev Dyn. 2004; 229(4):857-68. [DOI:10.1002/dvdy.10453] [PMID]

[17] Rios H, Koushik SV, Wang H, Wang J, Zhou HM, Lindsley A, et al. Periostin null mice exhibit dwarfism, incisor enamel defects, and an early-onset periodontal disease-like phenotype. Mol Cell Biol. 2005; 25(24):11131-44. [DOI:10.1128/MCB.25.24.11131-11144.2005] [PMID] [PMCID]

[18] Rios HF, Ma D, Xie Y, Giannobile WV, Bonewald LF, Conway SJ, et al. Periostin is essential for the integrity and function of the periodontal ligament during occlusal loading in mice. J Periodontol. 2008; 79(8):148090. [DOI:10.1902/jop.2008.070624] [PMID] [PMCID]

[19] Balli U, Keles ZP, Avci B, Guler S, Cetinkaya BO, Keles GC. Assessment of periostin levels in serum and gingival crevicular fluid of patients with periodontal disease. J Periodontal Res. 2015; 50(6):707-13. [DOI:10.1111/jre.12254] [PMID]

[20] Horiuchi K, Amizuka N, Takeshita S, Takamatsu H, Katsuura M, Ozawa $\mathrm{H}$, et al. Identification and characterization of a novel protein, periostin, with restricted expression to periosteum and periodontal ligament and increased expression by transforming growth factor beta. J Bone Miner Res. 1999; 14(7):1239-49. [DOI:10.1359/jbmr.1999.14.7.1239] [PMID]

[21] Sidhu SS, Yuan S, Innes AL, Kerr S, Woodruff PG, Hou L, et al. Roles of epithelial cell-derived periostin in TGF-beta activation, collagen production, and collagen gel elasticity in asthma. Proc Natl Acad Sci U S A. 2010; 107(32):14170-5. [DOI:10.1073/pnas.1009426107] [PMID] [PMCID]

[22] Nakajima M, Honda T, Miyauchi S, Yamazaki K. Th2 cytokines efficiently stimulate periostin production in gingival fibroblasts but periostin does not induce an inflammatory response in gingival epithelial cells. Arch Oral Biol. 2014; 59(2):93-101. [DOI:10.1016/j.archoralbio.2013.10.004] [PMID] 
This Page Intentionally Left Blank 